\title{
Minute Times Femtogram Per Milliliter Per Kilogram
}

National Cancer Institute

\section{Source}

National Cancer Institute. Minute Times Femtogram Per Milliliter Per Kilogram. NCI

Thesaurus. Code C112335.

Minutes times femtograms per milliliter, divided by kilograms. 